\title{
Successful Percutaneous Nephrolithotomy Using Thoracic Paravertebral Block as the Sole Surgical Anesthesia: Cases Report
}

Tanan Bejrananda, M.D. ${ }^{1}$, Jatuporn Pakpirom, M.D. ${ }^{2}$

'Urology Unit, Department of Surgery, Faculty of Medicine, Prince of Songkla University, Hat Yai, Songkhla 90110, Thailand. ${ }^{2}$ Department of Anesthesiology, Faculty of Medicine, Prince of Songkla University, Hat Yai, Songkhla 90110, Thailand.

Received 21 July 2020 • Revised 3 October 2020 • Accepted 17 October 2020 • Published online 24 March 2021

\begin{abstract}
:
In general, percutaneouse nepholithotomy (PCNL) is usually done under general anesthesia or occasional epidural anesthesia. We reported three renal calculi cases, who had successful PCNL surgery using ultrasound-guided multiple injections for thoracic paravertebral block at the T8, T10, and T12 levels with 20 milliliter of $0.5 \%$ levobupivacaine for renal surgery on a unilateral side. Sensory blockade was evaluated at 30 minutes using pinprick sensation before starting the operation. Sensory blockade on the unilateral side from level T8 to L1 was achieved in all patients. During the procedure, dexmedetomidine infusion at 0.2 to 0.6 microgram per kilogram per hour was administered for patient sedation. All three cases reported no pain in the recovery room and mild pain on the first and second postoperative visits. There was no complication from both multiple thoracic paravertebral injections and PCNL surgery.
\end{abstract}

Keywords: percutaneous nephrolithotomy, regional anesthesia, thoracic paravertebral block, ultrasound-guided

Contact: Asst. Prof. Jatuporn Pakpirom, M.D.

Department of Anesthesiology, Faculty of Medicine, Prince of Songkla University,

Hat Yai, Songkhla 90110, Thailand.

E-mail: pakpirom013@gmail.com

ights reserved.

This is an open access article under the CC BY-NC-ND license

(http://www.jhsmr.org/index.php/jhsmr/about/editorialPolicies\#openAccessPolicy).

J Health Sci Med Res 2021;39(5):423-429 doi: $10.31584 /$ jhsmr.2021788 www.jhsmr.org 


\section{Introduction}

In general, percutaneous nephrolithotomy (PCNL) is performed under general anesthesia. However, neuraxial blocks; especially an epidural block, provide for both surgical anesthesia and postoperative analgesia, so it can be used as the sole anesthetic procedure for PCNL surgery. ${ }^{1}$ For some patients, general anesthesia is not a preferred technique, and a neuraxial block should be avoided. An thoracic paraverterbral block (TPVB) is therefore an altenative choice to provide surgical anesthesia in PCNL surgery. ${ }^{1}$ TPVB is the injection of a local anesthetic drug into the paravertebral space (PVS) where the spinal nerves are seated and provides segmental unilateral sensory and motor blockade. Previous literature reports found that injection into the PVS provides effective postoperative analgesia after thoracotomy ${ }^{2}$, breast cancer surgery ${ }^{3}$, urological procedures ${ }^{4}$, and unilateral multiple rib fractures. ${ }^{5}$ In many studies, regional anesthesia established some advantages, such as early ambulation, faster recovery from surgery, less analgesic requirements, faster hospital discharge, and less postoperative pain. ${ }^{6}$ Compared to general anesthesia, TPVB is just as effective, requires less dosage, offers stable hemodynamic and pulmonary functions, normal contralateral myodynamia, and fewer adverse events. ${ }^{6,7}$ A previous study showed that multilevel TPVB from T10 to L1 was feasible in PCNL surgery. ${ }^{8}$ This study performed multiple TPVBs at each segment of the vertebrae (T10, T11 and T12), using a conventional technique. An ultrasound scan was only used to locate the transverse process of the spine, and then the loss of resistance technique was applied. ${ }^{8}$ However, there are no published reports using real-time multi-segment ultrasound guidance at alternating levels for TPVB as the main surgical anesthesia in PCNL surgery. Therefore, we performed multilevel TPVB at alternating segments (i.e. T8, T10, and T12) using real-time ultrasound guidance to unilateral both somatic and visceral pain sensation from the PCNL procedure. We believe that multilevel TPVB, at
T8, T10 and T12, is an alternative technique to provide surgical anesthesia for PCNL surgery in patients having contraindication of neuraxial block, or when there is a high risk in the use of general anesthesia.

Three patients were admitted in August 2019 in Songklanagarind Hospital to have PCNL surgery. A computerized tomography scan of the kidneys, ureters, and bladder (KUB) was done to confirm both the location and size of the stones. Written informed consent for PCNL procedures under TPVB was acquired from all three patients and patients were preoperative-visited by the anesthesiologist to prepare and give advice for the TPVB surgery.

\section{TPVB technique}

Intravenous access was established when the patient arrived in the operating room. Continuous monitoring included pulse oximetry, electrocardiography, and noninvasive blood pressure. Midazolam 1-2 milligram (mg) and ketamine $10 \mathrm{mg}$ were administered to facilitate patient comfort during the procedure. An oxygen facemask was applied and $\mathrm{CO}_{2}$ monitoring was commenced. Before turning the patient to the lateral position, the side of the operation was confirmed with the patient and surgeon. The patient was then turned to the lateral decubitus position with the surgical side kept upwards with the neck and hip slightly flexed (Figure 1A). The thoracic intervertebral levels were identified using the palpation technique and levels T8, T10, and T12 were marked on the patient's skin.

An ultrasound pre-scan was done to optimize thoracic paravertebral space (TPVS) visualization using a low frequency (3-6 megahertz) curvilinear transducer (Figure 1B). To perform three injections of TPVB (3m-TPVB) at the articular processes ${ }^{9}$, the ultrasound transducer was initially placed for a paramedian transverse scan [2 centimeter (cm) lateral from the midline] to visualize the transverse process. After that, the ultrasound transducer was moved slightly 
caudally until the window of the transverse process is disappeared. At that level the PVS has the widest visualization of space (Figure 1C). ${ }^{9}$ The block procedure was done under sterile conditions and the ultrasound transducer was also covered with a sterile drape. 3m-TPVB at levels T8, T10, and T12 was performed under real-time ultrasound guidance starting from the T8 to the T10 and T12 levels. Once the PVS was clearly identified, the patient's skin was anesthetized with $1.0 \%$ lidocaine without adrenaline before block needle insertion. Thereafter, the block needle (SonoTAP, Size $22 \mathrm{G} \times 80 \mathrm{~mm}$ ) was inserted and advanced from lateral to medial and posterior to anterior direction under real-time ultrasound guidance (Figure 1D-E). Once the needle tip was located inside the PVS, normal saline was injected to confirm the right position of the needle by visualizing anterior compression of the parietal pleura and widening of PVS during injection (Figure 1E-F). Six to $7 \mathrm{ml}$ of $0.5 \%$ levobupivacaine with adrenaline 5 microgram per milliliter was then incrementally injected at each level. The total amount of levobupivacaine was 20 milliliter.
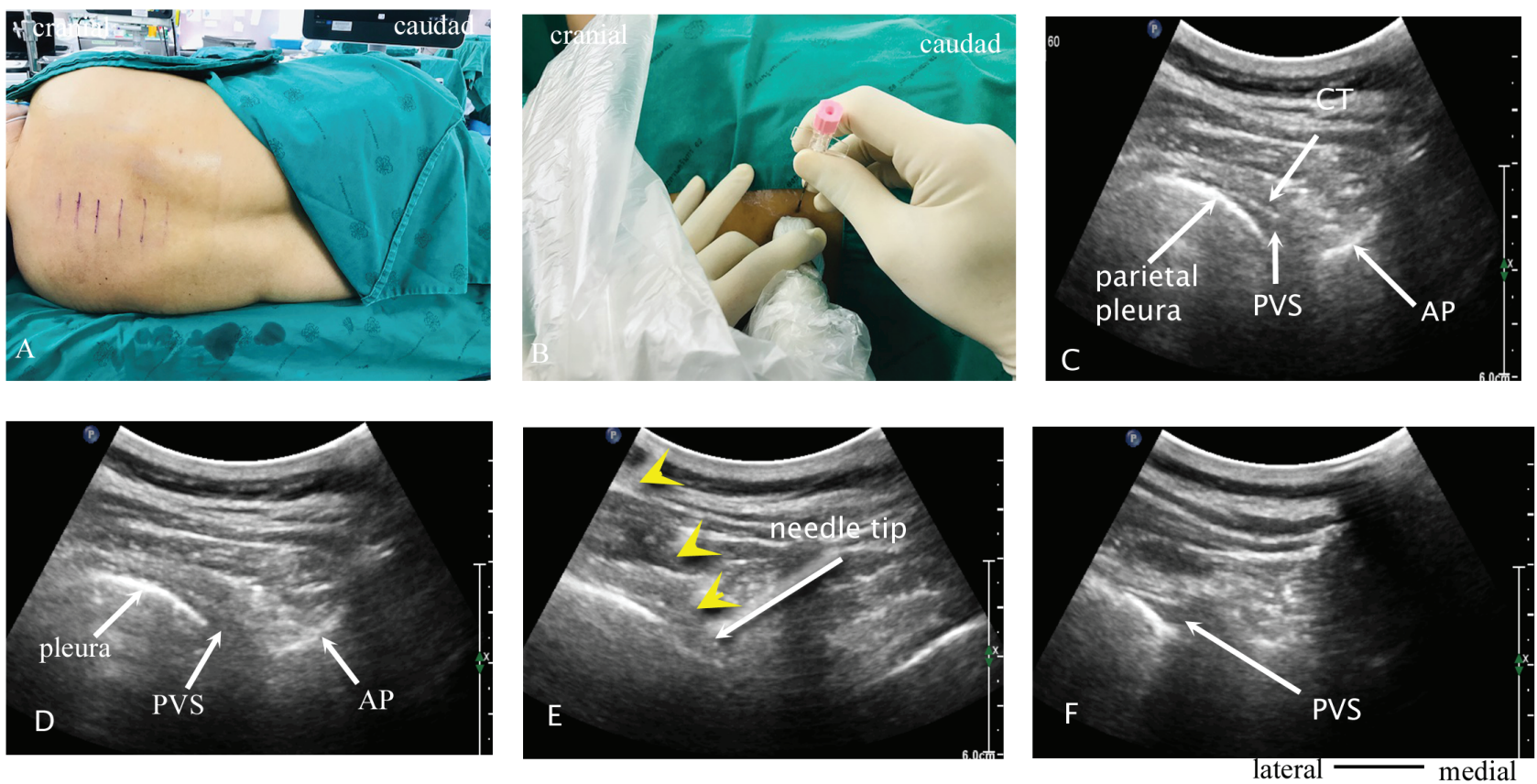

$\mathrm{CT}=$ costotransverse ligament, $\mathrm{PVS}=$ paravertebral space, $\mathrm{AP}=$ articular process

Figure 1 The TPVB technique is demonstrated including the patient position, ultrasonic anatomy of TPVB, and ultrasound-guided 3m-TPVB. (A) The patient is placed in the lateral position with the side to be blocked in the uppermost. (B) The curvilinear transducer is placed in the paramedian transverse scan direction to show the needle insertion from the posterolateral to anteromedial direction. (C) The ultrasound image of the TPVS at the level of the articular process. (D) Parietal pleura is anteriorly compressed and widening of the PVS during local anesthetic injection is shown. (E) The direction of the needle and position of needle tip is shown. The yellow arrows indicate the needle (F) After local anesthetic injection, widening of the PVS is shown 
Following the procedure for complete paravertebral block, sensory blockade was assessed in the three patients using pinprick sensation. All three cases achieved sensory block from T8 to L1 at 30 minutes after TPVB. During the PCNL surgery, standard monitoring, including electrocardiogram, noninvasive blood pressure monitoring, and pulse oximeter, was applied and an oxygen facemask at 5 liters per minute was also administered. The end tidal $\mathrm{CO}_{2}$ and respiratory rate were continuously monitored to detect apnea or airway obstruction. During surgery, intravenous infusion of dexmedetomidine $0.2-0.6$ microgram per kilogram per hour was started for conscious sedation, and fentanyl and/or ketamine intravenous bolus were also given to supplement the patient's requirements.

\section{PCNL technique}

Before starting the PCNL procedure, 5.0\% lidocaine gel was injected into the urethra for topical anesthesia. The patients were then placed in the lithotomy position and retrograde ureter catheter insertion was initiated using cystoscopy. The patients were asked to turn to the prone position by themselves with minimal support from the operating room staff personnel (Figure 2A-B). Renal access was established under fluoroscopic guidance. Percutaneous renal puncture was done directly through the fornix to the target stone. After urine flow, a guidewire was then inserted through the needle into the renal pelvis for the guidance of dilators and a metallic dilator was used to dilate the percutaneous tract until 27 French $(\mathrm{Fr})$ and an Amplatz sheath 26 Fr was inserted. The nephroscope was passed through the metallic dilator into the calyx and the renal stone was disintegrated using the lithotripter shock pulse. After stone removal was completed, a double $\mathrm{J}$ stent was inserted into the ureter without nephrostomy and it was removed at 6 weeks after surgery. The procedure was performed by a single, experienced surgeons.
The patients were asked to report a perioperative pain score using a visual analogue scale of 0-10. Intraoperative hemodynamic parameters, nausea, vomiting, sedation, itching, and side effects were collected. Postoperative recovery profiles; including postoperative pain score and analgesic drug requirement, were evaluated on postoperative days one and two.

\section{Case description}

Patient no. 1: A 67-year-old male, American Society of Anesthesiologist (ASA) physical status II, had a $2.3 \mathrm{~cm}$ left lower pole calyceal calculus. His height and weight were $160.5 \mathrm{~cm}$ and $60.2 \mathrm{~kg}$. After careful discussion, the patient and his family were informed and consented to have a PCNL under ultrasound-guided 3m-TPVB. A successful stone free procedure was performed in 80 minutes without any complications. The patient reported no pain in the recovery room and mild pain during postoperative day one (Figure 2C-D).

Patient no. 2: A 70-year-old female, ASA physical status III, had bilateral renal calculi. One year prior, she underwent right PCNL. A left staghorn stone was scheduled for PCNL. Her height and weight were $163 \mathrm{~cm}$ and $60 \mathrm{~kg}$. The operation was successful using ultrasound-guided $3 \mathrm{~m}-\mathrm{TPVB}$ and the overall procedure took 90 minutes. She reported mild pain during the trocar dilatation for renal access. Thus, she received a supplement of a low-dose propofol infusion. She reported no pain in the recovery room and moderate pain during postoperative day one (Figure 2E-F).

Patient no. 3: A 47-year-old male, ASA physical status II, who had a right renal calculus underwent right PCNL. His height and weight were $170 \mathrm{~cm}$ and $60 \mathrm{~kg}$. We performed the same technique using ultrasound-guided $3 m-T P V B$. The operation was uneventful and it took around 160 minutes. He was pain-free immediately following surgery, but had mild pain during postoperative visit on day one (Figure 2G-H). 

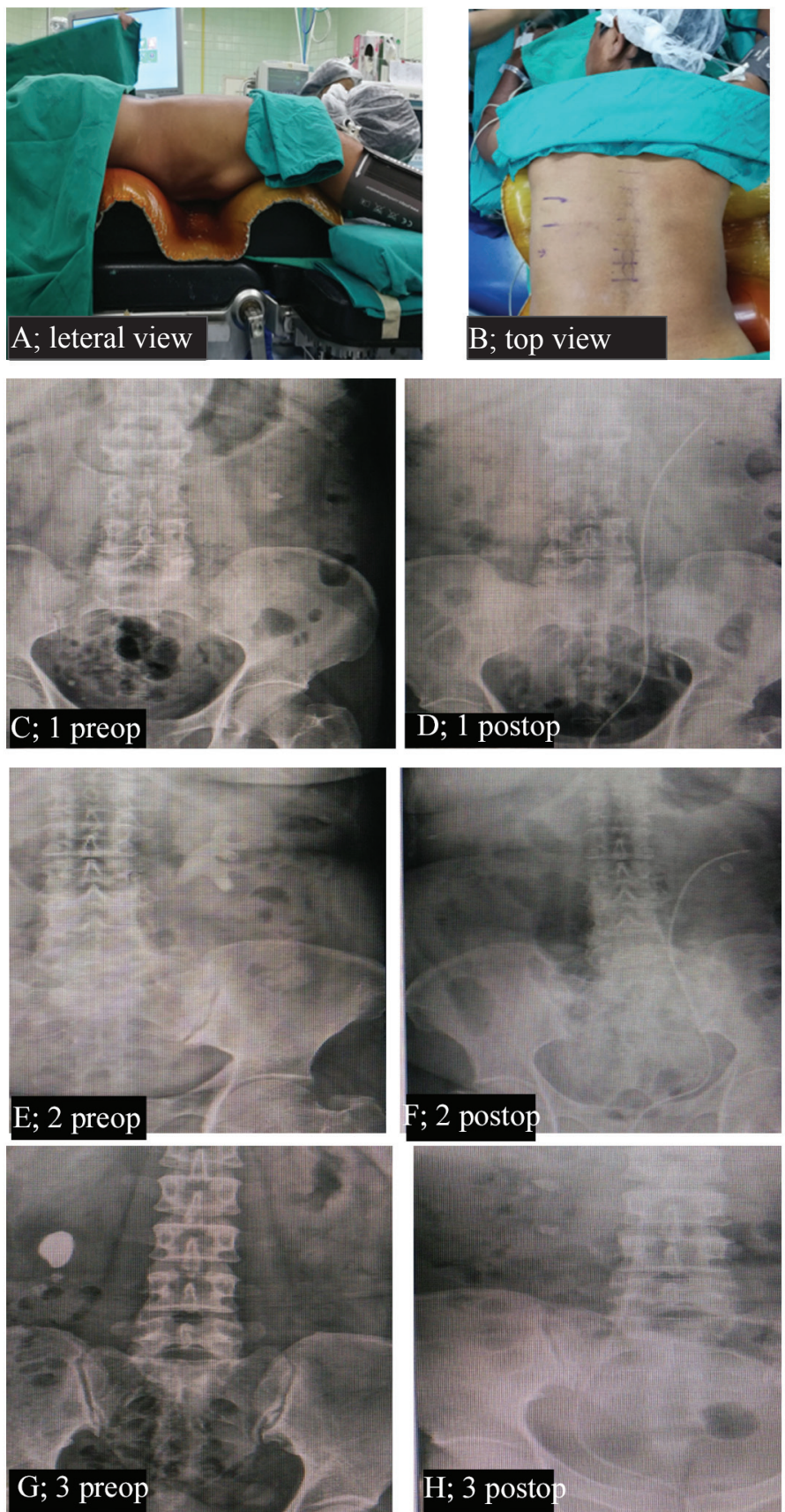

Figure 2 Positioning of the patient during surgery and a KUB plane film before and after PCNL are demonstrated. (A) The patient is in the lateral view position. (B) This is the top view of the patient. (C), (E), and (G) show KUB X-rays before the PCNL procedure in cases 1, 2, and 3, respectively. $(D),(F)$, and $(H)$ show KUB X-rays after the PCNL procedure in cases 1,2 , and 3 , respectively. 


\section{Discussion}

All three patients underwent successful PCNL surgery using TPVB as the sole anesthesia without any complications during and after surgery. TPVB is usually performed to provide postoperative analgesia that is combined with general anesthesia. Multilevel TPVB can be used as surgical anesthesia in major breast cancer surgery with conscious sedation. ${ }^{9}$ However, there is limited information using TPVB as the sole anesthesia for the PCNL procedure. Renal pain sensation is transmitted through nerves originating from levels $T 10$ to $L 1$, while pain sensation from the ureter is transmitted to the spinal from T10 to L2. ${ }^{10}$ Based on an anatomical aspect, complete blockade of the unilateral spinal nerves from $\mathrm{T} 10$ to $\mathrm{L} 2$ could provide adequate surgical anesthesia for unilateral PCNL. Thus, in TPVB where the local anesthetic is deposited at the most proximal part of the spinal nerve next to the intervertebral foramen, complete sensory blockade from T10 to L2 should be feasible for unilateral PCNL surgery.

The TPVS is a potential space that is continuous at all thoracic levels at the anteromedial corner and it contains the spinal nerves emerging from the intervertebral foramen. ${ }^{11}$ However, a single injection at only one level of the TPVS may limit the spread of the local anesthetic drug and compromise extension of somatic blockade. ${ }^{12,13}$ Therefore, multiple injections at more than one level are needed in TPVB for complete sensory blockade for surgical anesthesia. $^{9}$

In the present case report, we performed separate injections at three levels of the TPVS (T8, T10, T12) to ensure adequate spreading of the local anesthetic and complete sensory blockade from level T10 to level L2 for surgical anesthesia in PCNL. In addition, we injected about $7 \mathrm{ml}$ of local anesthetic at each level for a total of $20 \mathrm{ml}$ of $0.5 \%$ levobupivacaine. Previous studies reported that every 3 to $4 \mathrm{ml}$ of local anesthetic provides sensory blockade at one level. ${ }^{14,15}$ It was reported there may be a connection of the TPVS to the lumbar level. ${ }^{16}$ Following injection of the local anesthetic at the $12^{\text {th }}$ TPVS, we found that sensory blockade extended to the L1 level which indicated local anesthetic spread from the TPVS to the lumbar level.

The ultrasound-guided TPVB is a special procedure that requires the technical and ultrasound skills of an anesthesiologist to perform this block. Some techniques for ultrasound-guided lumbar PVB, known by stimulator guidance or loss of resistance, have been used to identify the PVS and the nerves. ${ }^{17}$ We routinely use ultrasound guidance for both thoracic and lumbar PVB. The important point is to identify the transverse process, articular process, costotransverse ligament, and the pleura because those structures are anatomically related to the TPVS. Moreover, it is necessary to track advancement of the needle under real-time ultrasound guidance and confirm proper injection at the TPVS by visualizing displacement of the parietal pleura during the injection of either normal saline or local anesthetic.

For PVB, locating the level of intervertebral space usually is performed by palpation at the level of the vertebrae which has a tendency to locate the wrong level. ${ }^{13,16,18}$ One recommended method for identification of the exact level of the transverse process is to identify the $12^{\text {th }}$ rib and then using a counting up approach to the wanted vertebral level. After this the transducer is medially moved to the midline, to locate the junction between the rib and the transverse process. There are 2 points for considering the $12^{\text {th }}$ rib these are: (1) it is a floating rib, hence when we use US scans laterally the bony structure of the rib will disappear; (2) To differentiate the $12^{\text {th }}$ rib from the L1 transverse process, the bony structure (hyperechoic line with acoustic shadow on US imaging) of the $12^{\text {th }}$ rib must be longer than the $L 1$ transverse process. 


\section{Conclusion}

Multilevel TPVB is feasible as the sole anesthesia for unilateral PCNL surgery and demonstrated a faster recovery from the surgery with a lower pain score during recovery and during the postoperative period. However, further study is needed to determine the safety and efficiency of 3m-TPVB for surgical anesthesia in patients undergoing PCNL.

\section{References}

1. Li C, Song C, Wang W, Song C, Kong X. Thoracic paravertebral block versus epidural anesthesia combined with moderate sedation for percutaneous nepholithotomy. Med Princ Pract 2016;25:417-22.

2. D'Ercole F, Arora H, Kumar PA. Paravertebral block for thoracic surgery. J Cardiothorac Vasc Anesth 2018;32:915-27.

3. Karmakar MK, Samy W, Li JW, Li A, Chan WC, Chen PP, et al. Thoracic paravertebral block and its effects on chronic pain and health-related quality of life after modified radical mastectomy. Reg Anesth Pain Med 2014;39:289-98.

4. Ak K, Gursoy S, Duger C, Isbir AC, Kaygusuz K, Ozdemir Kol I, et al. Thoracic paravertebral block for postoperative pain management in percutaneous nephrolithotomy patients: a randomized controlled clinical trial. Med Princ Pract 2013;22: 229-33.

5. Karmakar MK, Ho AM. Acute pain management of patients with multiple fractured ribs. J Trauma 2003;54:615-25.

6. Lonnqvist PA, Hildingsson U. The caudal boundary of the thoracic paravertebral space. A study in human cadavers. Anaesthesia 1992;47:1051-2.

7. Ozkan D, Akkaya T, Comert A, Balkc N, Ozdemir E, Gumus H, et al. Paravertebral block in inguinal hernia surgeries: two segments or 4 segments? Reg Anesth Pain Med 2009;34: 312-5.

8. Yang H, Yu X, Hu J, Peng E, Li C, Cui L, et al. Usage of Multilevel Paravertebral block as the Main Anesthesia fo Mini-invasive PCNL: Retrospective Review of 45 cases with Large Stones. Urol Int 2017;99:326-30.

9. Pangthipampai P, Karmakar MK, Songthamwat B, Pakpirom J,
Samy W. Ultrasound-guided multilevel thoracic paravertebral block and its efficacy for surgical anesthesia during primary breast cancer surgery. J Pain Res 2020;13:1713-23.

10. Malhotra V, Sudheendra V, O'Hara J, Diwan S. Anesthesia and the renal and genitourinary system. In: Miller RD editor. Miller's Anesthesia. $7^{\text {th }}$ ed. Philadelphia: Churchill Living Stone; 2010; p.450-9.

11. Krediet AC, Moayeri N, Van Geffen GJ, Bruhn J, Renes S, Bigeleisen PE, et al. Different approaches to ultrasoundguided thoracic paravertebral block: an illustrated review. Anesthesiology 2015;123:459-74.

12. Saito T, Den S, Cheema SP, Tanuma K, Carney E, Carlsson C, et al. A single-injection, multi-segmental paravertebral block-extension of somatosensory and sympathetic block in volunteers. Acta Anaesthesiol Scand 2001;45:30-3.

13. Marhofer D, Marhofer P, Kettner SC, Kettner SC, Fleischmann E, Prayer D, et al. Magnetic resonance imaging analysis of the spread of local anesthetic solution after ultrasound-guided lateral thoracic paravertebral blockade: a volunteer study. Anesthesiology 2013;118:1106-12.

14. Cheema SP, Ilsley D, Richardson J, Sabanathan S. A thermographic study of paravertebral analgesia. Anaesthesia 1995; 50:118-21.

15. Chen H, Liao Z, Fang Y, Niu B, Chen A, Cao F, et al. Continuous right thoracic paravertebral block following bolus initiation reduced postoperative pain after right- lobe hepatectomy: a randomized, double-blind, placebo-controlled trial. Reg Anesth Pain Med 2014;39:506-12.

16. Karmakar MK, Gin T, Ho AM. Ipsilateral thoraco-lumbar anaesthesia and paravertebral spread after low thoracic paravertebral injection. Br J Anaesth 2001;87:312-6.

17. Naja Z, Al Tannir M, Naccache N, Rajab ME, Sayyid KA, Ziade $F$, et al. General anaesthesia combined with bilateral paravertebral blockade (T11-T12-L1) vs general anaesthesia for bilateral varicocelectomy. A randomized double-blind clinical trial. J Med Liban 2011;59:126-30.

18. Pangthipampai $P$, Karmakar MK, Songthamwat B, Pakpirom J. The accuracy of $7^{\text {th }}$ cervical spinous process identification using ultrasound transverse scan and parasagital scan compared to palpation technique. J Med Assoc Thai 2017;100:S173-9. 